\title{
Das Problem des Wesens und der Entstehung des Gefïhlslebens.
}

\author{
Von \\ Dr. Max Serog.
}

(Eingegangen am 28. Oktober 1911.)

In der modernen Psychologie, speziell der deutschen, nimmt das Problem des Gefühlslebens nur einen recht geringen Raum ein den zahlreichen und eingehenden Untersuchungen gegenüber, in denen man die anderen Erscheinungen des Seelenlebens, vor allem die Empfindungs- und Denkvorgänge zu erforschen suchte. Vielleicht mag das zum Teil im allgemeinen Charakter der Zeit seine Ursache haben und im Zusammenhang stehen mit einer gewissen Utberschätzung des Rein-Intellektuellen, wie sie der letztvergangenen Epoche wohl eigen war. Der wesentliche Grund aber, warum sich die Psychologie mit dem, was wir als "Gefühlsleben, Affekte, Gemütsbewegungen“ bezeichnen, nur erst so wenig beschäftigt hat, liegt darin, daß bei dem Dunkel, das hier noch herrscht, die Grundlage überhaupt fehlt, auf der eine Erforschung der Gefühlsvorgänge sich aufbauen könnte. Denn worin das Wesen dessen besteht, was wir als „Gefühl" bezeichnen, darauf gibt es bis heute noch keine einzige befriedigende Antwort und es ist bezeichnend, da $\beta$ keine einzige der verschiedenen Gefühlstheorien eine allgemeine Anerkennung hat finden können.

Jedenfalls steht man dem Wesen der Gefühlsvorgänge heute noch ganz anders gegenüber wie etwa dem der intellektuellen Funktionen. Bei der Erforschung der intellektuellen psychischen Funktionen ging man von der Tatsache der Vorstellungsverknüpfung aus. Die „Assoziationspsychologie" zeigte, daß sich die ganze intellektuelle Tätigkeit, auch in ihren höchsten und kompliziertesten Formen, auf die einfache Verknüpfung, die „Assoziation“ von Vorstellungen zurückführen läßt. Wenn nun auch die Anschauung der sog. reinen Assoziationspsychologie, nach der eine derartige Verknüpfung die ausschließ. liche Grundlage unseres intellektuellen Lebens bildet, nicht durchweg geteilt wird, so wird doch ihre wesentliche Rolle jedenfalls allgemein anerkannt. Nur auf der Grundlage dieser allgemein anerkannten Anschauung aber ist eine derartige eingehende Erforschung der Denkvorgänge, wie wir sie heute schon besitzen, und somit ein tieferer Einblick ịn diese Seite des seelischen Geschehens möglich gewesen.

So sehr nun auch die Denkvorgänge durch die Assoziationspsychologie unserem psychologischen Verständnis näher gebracht wurden, so 
wenig hat auch sie für das Gefühlsproblem eine befriedigende Lösung geben können. Dadurch, daß die Assoziationspsychologie sozusagen nur von der einen Seite an die Psyche heranging und zu sehr nur das intellektuelle psychische Geschehen berücksichtigte, wurde sie der eminenten psychischen Bedeutung des Gefühlslebens nicht gerecht. Was im speziellen gegen die Gefühlstheorie der Assoziationspsychologie eingewendet werden muß, soll später noch erörtert werden.

Um hier zunächst kurz auf die hauptsächlichsten der sonstigen neueren Gefühlstheorien einzugehen, so sei zuerst die James-Langesche Theorie erwähnt ${ }^{1}$ ).

Jeder Gefühlszustand geht bekanntlich mit gewissen körperlichen Erscheinungen (Veränderungen des Pulses, des Blutdruckes, der Atmung, bestimmten Muskelinnervationen usw.) einher, die gerade in den letzten Jahren öfters der Gegenstand sehr eingehender Untersuchungen gewesen sind (Lehmann, Berger, Weber $\left.{ }^{2}\right)$ ). Während diese körperlichen Veränderungen aber sonst eben als Folge- oder Begleiterscheinungen der Gefühlszustände aufgefaßt wurden, nimmt die JamesLangesche Theorie an, daß in ihnen und ausschließlich in ihnen das eigentliche Wesen der Gefühlsvorgänge zu suchen sei. Diese sind danach also nichts anderes als die Summe der Empfindungen bestimmter motorischer und vasomotorischer Innervationen; die körperlichen Veränderungen sind nicht eine Folge der Gefühlszustände, sondern ihre Ursache: „Wir weinen nicht, weil wir traurig sind, sondern wir sind traurig, weil wir weinen" (J $\left.\mathrm{J} \mathrm{mes}^{3}\right)$ ).

Nun ist es ja fraglos richtig, daß bei dem, was wir als irgendeinen „Gefühlszustand" erleben, auch die Empfindungen der dabei gleichzeitig auftretenden körperlichen Veränderungen eine Rolle spielen. Daß der ganze Gefühlszustand aber nichts weiter sei als die Summe dieser Empfindungen, trifft sicher nicht zu. Zunächst ist es überhaupt fraglich, ob wirklich jedem einzelnen der verschiedenen, doch schon bei gröbster Gruppierung sehr zahlreichen Gefühlszustände immer eine spezifische Kombination bestimmter körperlicher Erscheinungen entspricht. Die bisherigen Untersuchungen haben den Beweis dafür jedenfalls nicht geliefert. Weiter kommen aber die für bestimmte Gefühlszustände als charakteristisch angegebenen Innervationen auch ohne diese vor. Wenn aber die Gefühlszustände wirklich nichts anderes wären, als die Summe von Empfindungen gewisser motorischer Innervationen, müßten beim Eintreten dieser stets auch jene vorhanden sein.

1) Siehe besonders Lange, Die Gemütsbewegungen. Würzburg 1910.

2) Siehe besonders Lehmann, Die Hauptgesetze des Gefühlslebens, übersetzt von F. Bendixen, Leipzig 1892.

3) W. James, Psychologie, über.setzt von Dürr. Leipzig 1909. S. 376. 
Eine andere Gefühlstheorie wurde in neuerer Zeit von Forster ${ }^{1}$ ) aufgestellt. Ausgehend von den Schmerzpunkten und schmerzleitenden Bahnen sucht sie die „unangenehmen Gefühlstöne" durch Assoziation mit Schmerzempfindungen zu erklären, das Schwinden des Schmerzes erscheine uns dann als eine neue Empfindung, als „Lust“. Demgegenüber ist hervorzuheben, daß lustvolle Gefühlszustände etwas ebenso Positives sind als schmerzvolle, und daß es durchaus nicht angeht, jene einfach durch das Schwinden dieser erklären zu wollen. Auch ist die Annahme, daß, wenn z. B. das Kind an einer auch schwach bitteren oder salzigen Lösung leckt, neben den Geschmacksnerven stets auch Schmerznerven mit gereizt werden, eine ganz willkürliche und durch nichts bewiesene Bemerkenswert aber an dieser Theorie ist - und zwar gerade im Hinblick auf unsere späteren eigenen Ausführungen der hier unternommene Versuch, zur Lösung des Gefühlsproblems von bestimmten Empfindungen auszugehen. Bei den Einwänden gegen die Forstersche Theorie haben wir ganz davon abgesehen, daß diese Theorie die Einteilung der Gefühle in lust- und unlustbetonte zur Voraussetzung hat, eine Einteilung, die durchaus keine selbstverständlich gegebene, im Gegenteil in mancher Beziehung sogar anfechtbare ist.

Der gleiche Einwand ist also auch der Anschauung gegenüber zu erheben, daß die Gefühle mit dem jeweiligen Ernährungszustande der Nervenzelle in Zusammenhang stehen, und daß der normale Ernährungszustand der Nervenzellen mit Lustgefühlen, ein unzureichender Ernährungszustand der Zellen aber mit Unlustgefühlen verknüpft sei. Die Unmöglichkeit einer derartigen Annahme ergibt sich übrigens auch bereits aus der Tatsache, daß gerade die Zustände, bei denen eine Schädigung des Ernährungszustandes der Nervenzellen vorliegt, mit ausgesprochen lustvollen Gefühlen einhergehen. Gerade für die Erschöpfung sind manische und hypomanische Zustände charakteristisch, wie wir aus klinischen Beobachtungen wie experimentellen Untersuchungen wissen; und das gleiche gilt von manchen Intoxikationen, z. B. dem Alkohol.

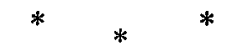

Geht man unvoreingenommen durch jede Theorie an das Gefühlsproblem heran und versucht, sich möglichst unbefangen darüber klar zu werden, was wir in irgendeinem Gefühlszustand psychisch erleben, so können wir folgendes feststellen.

Nehmen wir etwa das Gefühl der Freude. Wir haben dabei (wenn wir von den Empfindungen der gleichzeitig auftretenden körperlichen Erscheinungen, z. B. der Empfindung eines lebhafteren Herzschlages absehen) erstens eine Anzahl von Vorstellungen und Vorstellungsreihen,

1) Siehe Forster, Über die Affekte. Monatsschr. f. Psych. u. Neurol. 19, H. 3 u. 4 , 1906. 
etwa von den Tatsachen, die die Ursache der Freude sind, ihren möglichen Folgen usw., und zweitens eben das, was wir als „Gefühl der Freude" bezeichnen. Mehr können wir aber darüber nicht sagen. Die dabei in uns auftauchenden Vorstellungsreihen können wir weiter bis ins einzelne zergliedern, der gleichzeitig mit diesen Vorstellungen auftretende "Gefühlszustand" ist dagegen einer weiteren Analyse nicht zugänglich. Jedenfalls ist das, was wir psychisch erleben, wenn ein Gefühl uns beherrscht, und wenn eine Vorstellung in uns auftaucht, etwas, was der Selbstbeobachtung in toto verschieden und völlig unvergleichbar erscheint; daher hat man auch, seit es überhaupt eine Psychologie gibt, den grundsätzlichen Unterschied zwischen Denken und Fühlen stets aufrecht erhalten und sie als verschiedene seelische „Fähigkeiten“ einander gegenüber gestellt. Gerade in diesem Punkte nun, in der Inkommensurabilität der Gefühle, gemessen an Vorstellungen, liegt aber eben die Schwierigkeit, wenn nicht Unmöglichkeit ihrer psychologischen Analyse. Denn eine solche Analyse ist im Grunde doch ein Zurückführen auf Vorstellungen; schon dadurch, daß man diese Analyse nur in Worten geben kann, werden die Gefühlszustände zu Begriffen, also Vorstellungskomplexen und verlieren so ihr eigentliches Wesen. Von diesem Standpunkt aus gesehen ist auch eine Einteilung der Gefühle gewissermaßen ein Unding. Im besonderen ist die Einteilung in Lust- und Unlustgefühle deshalb abzulehnen; sie ist bereits etwas Abstrakt-Intellektualistisches, Reflektierendes, also etwas, was in den Gefühlen selbst nicht von vornherein gegeben, ihnen als solchen vielmehr fremd ist. Das nachträglich Abstrahierende ist schon in der Gegensätzlichkeit dieser Zweiteilung ersichtlich. Die sich dem naiven Bewußtsein zunächst bietenden Gefüblszustände zeigen durchaus nicht alle ausgesprochen einen entweder positiven oder negativen Charakter, es sei hier nur an die Wut erinnert.

Man sieht also: auf dem Wege einer psychologischen Analyse im eigentlichen Sinne, das heißt einer Zurückführung als komplex erkannter Gebilde auf einfache, wie wir sie bei den Denkvorgängen vornehmen können, und wie sie uns hier auch schon einen gewissen Einblick in diese Vorgänge verschafft hat, auf diesem Wege also kommen wir bei den Gefühlsvorgängen nicht weiter. Was wir hier analysieren können, sind immer und ausschließlich nur die mit den Gefühlen zusammen auftretenden Vorstellungskomplexe, die Gefühlszustände selbst aber bleiben ein Etwas, das wir zwar als solches immer wieder erleben, aber nicht weiter erklären können. Wohl aber können wir uns die Frage vorlegen, ob wir etwas Derartiges wie in den Gefühlszuständen nicht auch in anderen, vielleicht einfacheren psychischen Verhältnissen wiederfinden. Dabei drängt sich nun sofort die Tatsache auf, daß wir bei einer Reihe von Empfindungen, und vor allem bei den Hautsinnes 
empfindungen, ganz etwas Derartiges psychisch erleben wie bei den Gefühlszuständen. Das angenehme Gefühl, das ich etwa in einem wohltemperierten Bade habe, ist nichts prinzipiell Andersartiges als das angenehme Gefühl, das ich in einem Kreis mir gleichgestimmter, sympathischer Menschen empfinde. Am deutlichsten wohl treten die hier in Rede stehenden Beziehungen beim Schmerz zutage. Hier kann man geradezu zweifelhaft sein, ob der Schmerz im wesentlichen eine Empfindung oder ein Gefühlszustand ist ${ }^{1}$ ). Denn einerseits wissen wir, daß er, entsprechend den anderen Empfindungen, in besonderen Bahnen im Rückenmark und Gehirnstamm, wahrscheinlich sogar auch in der Peripherie lokalisiert ist, andererseits erscheint uns aber das, was wir an und in ihm psychisch erleben, den Gefühlszuständen viel näher verwandt zu sein als den anderen Empfindungen. Daß das der Fall ist, und daß in der Tat der Schmerz uns vielmehr etwas Gefühls- als etwas Empfindungsmäßiges zu sein scheint, geht schon daraus hervor, $\mathrm{da} ß$ der Schmerz für uns geradezu - nach Ble ulers Ausdruck -- zum „Prototyp aller negativen Affekte" geworden ist $\left.{ }^{1}\right)$. Ähnlich wie mit dem Schmerz ist es mit den anderen Hautsinnesempfindungen; auch sie besitzen sehr ausgesprochen das, was wir als "Gefühlskomponente" bezeichnen wollen. Wir wählen diesen Ausdruck, da er nur die geschilderte Tatsache wiedergibt, ohne zunächst darüber ein Urteil zu fällen, ob diese „Gefühlskomponente“ nur eine Eigenschaft der Empfindung (,Gefühlsbetonung“") oder etwas Andersartiges, zu der eigentlichen Empfindung Hinzukommendes ist. Diese Gefühlskomponente kommt nun zwar den Hautsinnesempfindungen in besonderem Maße, aber nicht ausschließlich zu. Auch bei den anderen Empfindungen finden wir sie, wenn auch bei den verschiedenen Sinnesgebieten in verschiedenem Grade. Das Durcheinanderwerfen der Begriffe "Empfindung" und "Gefühl", wie sie der älteren Psychologie eigen war, findet in dieser Gefühlskomponente der Empfindungen seine wohlbegründete Erklärung. Die laienhafte Psychologie gebraucht ja noch heute ,empfinden' und ,fühlen' in gleichem Sinn.

Betrachten wir nun die Empfindungen der anderen Sinnesgebiete auf den Grad ihrer Gefühlskomponente hin, so sehen wir, daß sie auch bei den Geruchs- und Geschmacksempfindungen noch sehr deutlich vorhanden ist. Dagegen tritt sie bei den sog. höheren Sinnen Gehör und Gesicht, mehr zurück, bei den Gesichtsempfindungen ist sie wohl kaum noch vorhanden. Bezeichnenderweise spricht man ja von Gehör und Gesicht auch als von den ,objektiven“ Sinnen. Die Empfindungen bilden sonach nach dem Grade ihrer Gefühlskomponente eine kontinuierliche Reihe, die von den Hautsinnesempfindungen ausgehend zu den Geruchs-, Geschmacks-, Gehörs- und schließlich Gesichtsempfindungen führt.

1) Vgl. Bleuler, Affektivität, Suggestibilität, Paranoia. Halle 1906. S. 9. 
In dem Maße aber, in dem in dieser Reihe die Gefühlskomponente der Empfindungen abnimmt, nimmt etwas anderes zu, nämlich die Möglichkeit der Reproduzierbarkeit der Empfindungen, also die Möglichkeit, zu Vorstellungen zu werden.

Die Empfindung eines Schmerzes können wir überhaupt nicht reproduzieren; die Vorstellung eines Schmerzes können wir nicht bilden, wir können immer nur die Schmerzempfindung aufs neue erleben. Ähnlich verhält es sich mit den anderen Hautsinnesempfindungen. Bei den Geschmacks- und Geruchsempfindungen ist eine Reproduktion jedenfalls nur sehr schwer, bei vielen Menschen sicher überhaupt nicht möglich. (Nach einer Statistik von Ribot ${ }^{1}$ ) konnten $60 \%$ der von ihm befragten Personen sich Gerüche vorstellen, aber meist nur in Verbindung mit den zugehörigen Gesichtsvorstellungen.) Es sei u. a. hier darauf hingewiesen, daß da, wo man z. B. im Traum Geschmacks- oder Geruchsvorstellungen zu haben glaubt, wie sich bei genauerem $\mathrm{Zu}$ sehen bald ergibt, doch nur stets Gesichtsvorstellungen vorhanden waren. Die Gesichts- und dann die Gehörsempfindungen sind jedenfalls die am leichtesten reproduzierbaren. Mit der Möglichkeit der Reproduzierbarkeit der Empfindungen hängt nun aber etwas anderes eng zusammen, nämlich ihre Klarheit und Bestimmtheit, das, was wir als das „Objektive" an ihnen empfinden. Je größer die Möglichkeit der Reproduzierbarkeit einer Empfindung ist, um so klarer und bestimmter erscheint uns die betreffende Empfindung. In dem Maße also, in dem in der obigen Reihe die Gefühlskomponente zurücktritt, die Leichtigkeit der Reproduzierbarkeit aber zunimmt, werden die Empfindungen auch schärfer, klarer, bestimmter, es tritt das an ihnen mehr hervor, was wir als ihren „Inhalt" bezeichnen. Daher schreiten also die Empfindungen der einzelnen Sinnesgebiete auch - wie Jodl es ausgedrückt hat _ ,,in sukzessiven Ubergängen vom Inhaltsarmen, aber Gefühlskräftigen zum Inhaltsreichen, aber Gefühlsschwachen " ${ }^{2}$ ) fort.

Die hier aufgestellte Behauptung, daß die Klarheit und Bestimmheit des Inhalts einer Empfindung durch die Leichtigkeit ihrer Reproduzierbarkeit bedingt ist, mag vielleicht Widerspruch hervorrufen; man kann darauf hinweisen, daß es der oberflächlichen Betrachtung doch eher umgekehrt erscheint. Dem ist entgegen zu halten, daß das, was man rein tatsächlich hier konstatieren kann, zunächst nichts weiter als diese gegenseitige Abhängigkeit ist: das Inhaltsvolle und die Reproduzierbarkeit einer Empfindung nehmen auf den verschiedenen Sinnesgebieten in gleichem Maße zu und ab. Ich kann mir nun aber unter der Reproduzierbarkeit einer Empfindung psycho-physiologisch viel eher etwas vorstellen, als etwa unter dem Objektiven einer Empfindung.

1) Ribot, Psychologie der Gefühle, übers. v. Chr. Ufer. Altenburg 1903. S. 182 ff.

2) $\mathrm{J}_{\mathrm{O}}$ d], Lehrbuch der Psychologie 2. Stuttgart und Berlin 1908. S. 28. 
Insbesondere ist für die obige Anschauung noch folgendes anzuführen. Etwas Objektiveres, Inhaltsreicheres wie es etwa die Gehörs- und besonders die Gesichtsempfindungen anderen Arten von Empfindungen gegenüber besitzen, haben diese Empfindungen offenbar für uns dadurch, daß sie eine besonders große Tendenz haben, zu Vorstellungen zu werden. Denn einen ,Inhalt' gewinnen sie ja für uns erst durch die Verknüpfung mit anderen Vorstellungen. Zu Vorstellungen werden können die Empfindungen aber nur in dem Grade, in dem sie repro. duzierbar sind.

Während also die schwer oder gar nicht reproduzierbaren Empfindungen sich nicht untereinander assoziieren, ordnen sich die aus den leicht reproduzierbaren Empfindungen hervorgegangenen Vorstellungen $\mathrm{zu}$ zusammengesetzten Gebilden, so die Gesichtsvorstellungen zu Objekten.

So wurden also die leicht reproduzierbaren, aber eine nur geringe Gefühlskomponente besitzenden Gehörs- und Gesichtsempfindungen zur Grundlage unseres Vorstellungslebens, unserer Ideenassoziation, so baute sich auf ihnen unser intellektuelles Leben, unsere „Erkenntnis“ auf. Die objektivsten und leicht reproduzierbarsten und gleichzeitig gefühlsärmsten Empfindungen, die Gesichtsempfindungen, haben vermutlich im Anfang dieser intellektuellen Entwicklung die Hauptrolle gespielt. Unser heutiges Denken ist ja eng an die Sprache geknüpft, ein koordiniertes und damit begriffliches Denken ist ja überhaupt erst möglich geworden, als das Sprechen begann und damit motorische Elemente in der Funktion des Denkens eine Rolle zu spielen anfingen. Früher aber, bevor es eine Sprache gab, war das „Denken“ — wenn man diesen Ausdruck dafür überhaupt schon gebrauchen will - wahrscheinlich im wesentlichen ein visuelles, $d$. $h$. eine assoziative Verknüpfung von Gesichtsvorstellungen, wie wir es bei den höchststehenden Tieren wohl annehmen können und bei uns heute noch im Traume finden (daher die Menge der Erlebnisse innerhalb kurzer Zeit und somit die scheinbare Geschwindigkeit des Gedankenablaufs im Traum).

Wenn man übrigens die verschieden starken Gefühlskomponenten der Empfindungen in der oben gegebenen Reihe teleologisch betrachten wollte, so könnte man sich vielleicht vorstellen, daß eine solche Gefühlskomponente auf einer früheren Stufe der Entwicklung allen Empfindungen in gleichem Grade eigen war, in dem Maße aber, wie die Empfindungen durch die Möglichkeit der Reproduzierbarkeit die Fähigkeit erlangten, zu Vorstellungen zu werden, ging diese Gefühlskomponente verloren, indem das nun entstandene „Gedächtnis" die Funktion der ursprünglich direkt an die Empfindung gebundenen Gefühlskomponente übernahm, als Schützer des Organismus zu dienen. In jedem Falle aber erscheint dieser Zusammenhang zwischen Möglichkeit 
der Vorstellungsbildung und Verlieren der Gefühlskomponente gerade auch für das Verständnis höherer und komplizierter psychischer Erscheinungen recht beachtenswert.

Wir sahen in unserer Empfindungsreihe die Empfindungen vom schwer Reproduzierbaren, aber Gefühlskräftigen zum leicht Reproduzierbaren, aber Gefühlsschwachen fortschreiten. Wir sahen ferner, wie die gefühlsarmen Empfindungen vermöge ihrer leichten Reproduzierbarkeit zur Grundlage unseres Vorstellungs-, unseres intellektuellen Lebens wurden. Die nur schwer oder gar nicht reproduzierbaren, aber besonders gefühlsstarken Empfindungen können nun einerseits eben wegen ihrer schweren Reproduziorbarkeit zum Vorstellungsleben zunächst keine Beziehung haben, andererseits ist ein besonderes Hervortreten der Gefühlskomponente das, was sie vor den leicht reproduzierbaren, objektiven Empfindungen auszeichnet. Die Frage liegt also sehr nahe, ob nicht, wie diese Empfindungen zum intellektuellen, jene zum affektiven psychischen Leken in besonderer Beziehung stehen. Es gibt nun auch eine Reihe v sn Tatsachen, aus denen hervorgeht, daß diese Empfindungen - wie ersichtlich, handelt es sich hier hauptsächlich um die Hautsinnesempfindungen - in vielfacher und enger Beziehung zu dem stehen, was wir als unser Gefühlsleben unserem Vorstellungsleben gegenüber stellen. Bemerkenswert sind hier schon die Hinweise, die die Sprache gibt, indem sie das gleiche Wort für die Affekte ebenso wie für die Hautsinnesempfindungen gebraucht: man ,fühlt" Schmerz, Mitleid, Freude, ebenso wie man eine Berührung, einen Nadelstich ,fühlt". Zwischen der Ausbildung der Hautsinnesempfindungen und des Gefühlslebens, zwischen der Sensibilität und der Affektivität besteht eine deutliche Parallelität. Affektiv Úbererregbare sind sehr oft auch hyperästhetisch. Bei affektverblödeten Idioten, auch Verbrechern finden wir häufig Anästhesie (Lombroso). Diejenige geistige Erkrankung, die speziell eine Abstumpfung der Affekte zur Folge hat, die Dementia praecox, zeigt sehr gewöhnlich auch eine Aufhebung der Schmerzempfindlichkeit, indem die normalerweise auf Schmerzreize eintretende Pupillenerweiterung hier ausbleibt. Dieselben Narcotica, die im Sinne einer Herabsetzung der Affektivität wirken, haben die gleiche Wirkung auch auf die Sensibilität.

Die Hautsinnesempfindungen sind also diejenigen Empfindungen, die in ihrer besonders ausgesprochenen Gefühlskomponente die deutlichsten Beziehungen zur Affektivität zeigen. Aus der allgemeinen Sensibilität heraus, die ja in der Stammesreihe die ursprünglichste Sinnesempfindung war, aus der sich erst später die anderen Empfin- 
dungen differenzierten, hat sich also die Affektivität entwickelt'1). Das Verhältnis der Sensibilität zur Affektivität ist ein ähnliches wie das der Sprache zum Intellekt: die Sensibilität ist, wenn auch nicht die einzige, so doch die wesentlichste, jedenfalls die ursprünglichste Komponente der Affektivität. Außer den Hautsinnesempfindungen sind dabei dann noch die inneren Empfindungen einschließlich der sexuellen Gefühle von besonderer Wichtigkeit. „Der primordiale Sinn, bei gewissen Tieren der einzige, ist der Tastsinn in Verbindung mit den inneren Empfindungen"2). Wir müssen somit einen gewissen Grad von Affektivität wohl schon bei den niedersten Tieren annehmen, bei denen von irgendeiner Art von Vorstellungsleben noch keine Rede sein kann.

Das Gefühlsleben ist also in seiner ursprünglichsten Form eine der ersten, primitivsten psychischen Fähigkeiten und geht der intellektuellen, der Vorstellungstätigkeit weit voran, und zwar sowohl ontogenetisch wie phylogenetisch. In seiner höchstentwickelten Form dagegen gehört das Gefühlsleben zu den letzterworbensten und feinstdifferenziertesten psychischen Funktionen, da es ein bereits hochentwickeltes Vorstellungsleben zur Voraussetzung hat. Denn unser „höheres Gefühlsleben“ kommt zustande durch eine eigenartige Verknüpfung der Affektivität mit Vorstellungen, die Affekte sind an Vorstellungskomplexe gebundene Affektivität (es sei an das obige Beispiel erinnert). Das in der Stammesreihe zuletzt Erworbene geht aber am leichtesten auch wieder verloren. Das Gefühlsleben kann, eben weil es etwas besonders Kompliziertes darstellt, am leichtesten durch irgendwelche Schädigung des Gehirns in Mitleidenschaft gezogen werden. So sehen wir z. B., daß bei organischen Gehirnerkrankungen (wie der Paralyse), die stets in ihrem Verlauf zu einer groben Schädigung der assoziativen Fühigkeiten führen, zunächst das höhere Gefühlsleben eine deutliche Einbuße erleidet, und zwar schon zu einer Zeit, wo die

1) Es sei hier darauf hingewiesen, daß auch bei der Entstehung des Ichgefühls, das uns ja als etwas von vornherein Gegebenes und nicht weiter Erklärbares erscheint, gerade Sensibilitätsempfindungen von wesentlichster Bedeutung sind. Nur durch sie kommen wir dazu, den eigenen Körper als etwas anderes als alle anderen Objekte, d. h. uns ihnen gegenüber als Subjekt zu empfinden. Wertvoll in dieser Hinsicht sind vor allem die Beobachtungen, die man in bezug auf die Fntstehung des Ichgefühls beim Kinde gemacht hat. „Nicht an dem Tage, an welchem das Kind zum ersten Male das Wort ,ich' statt seines Figennamens braucht, erwacht sein Ichgefühl - dieser Zeitpunkt variiert je nachdem die Angehörigen länger oder kürzer sich selbst und das Kind beim Namen, statt mit Fürwörtern nennen -, sondern das Ioh wird nach einer langen Reihe von Erfahrungen, hauptsächlich schmerzlicher Art, vom Nicht-Ich getrennt durch die Gewöhnung an die eigenen Körperteile" (Preyer, Die Seele des Kindes. Leipzig 1900. S. 391).

2) Ribot, 1. c. S. 137 
intellektuellen Funktionen noch völlig intakt sind. Die psychische Schädigung, die eine andere geistige Erkrankung, die Dementia praecox, setzt, bleibt häufig ausschließlich auf das Gefühlsleben beschränkt, die „Affektive Verblödung“ ist ja etwas für die Dementia praecox geradezu Charakteristisches.

Die Verknüpfung der Affektivität mit den Vorstellungen ist nun aber für unser intellektuelles Leben von besonderer Bedeutung. Durch sie wird eine über die bloße assoziative Aneinanderreihung von Vorstellungen hinausgehende Zusammenordnung derselben erreicht, ähnlich wie die Sensibilität die zweckmäßige Zusammenordnung, die Koordination von Bewegungen ermöglicht ${ }^{1}$ ). So wird die Affektivität die Vorbedingung zum geordneten, zielbewußten Denken und die „Aufmerksamkeit" eine der wichtigsten Äußerungen der Affektivität²). Wo die affektive Komponente der Vorstellungen fehlt, wie bei der Dementia praecox, leidet daher ihre Koordination, es kommt zu einer sozusagen ,,assoziativen Ataxie", zu Aufmerksamkeitsstörungen. Solche Aufmerksamkeitsstörungen finden wir bei der Dementia praecox in den Erscheinungen der Kontamination, der Sprachverwirrtheit, aber auch im normalen Leben können wir oft genug derartige Erscheinungen und ihr Zustandekommen durch Fehlen der Aufmerksamkeit beobachten $\left.{ }^{3}\right)$. Welcher Art ist nun aber diese Verknüpfung der Affektivität mit Vorstellungen, die eine für unser intellektuelles Leben so bedeutsame Rolle spielt?

Nach der Anschauung der Assoziationspsychologie treten Gefühle ausschließlich als Eigenschaften von Empfindungen und Vorstellungen, als „Gefühlstöne" auf; selbständig existierende Gefühle gibt es überhaupt nicht $\left.{ }^{4}\right)$. Das ist nun aber sicher nicht richtig; durchaus nicht ist in jedem Falle jeder emotionelle Zustand an einen intellektuellen gebunden. Vielmehr gibt es ein selbständiges Gefühlsleben, das seine Selbständigkeit schon dadurch beweist, daß es sich, wie wir sahen, vor dem intellektuellen Leben entwickelt. Nicht nur in der Stammesreihe, sondern auch beim Einzelindividuum sind die affektiven Fähigkeiten

1) Näheres über diese „Assoziative Koordination" siehe Serog, Psychische Störungen bei Stirnhirntumoren und die Beziehungen des Stirnhirns zur Psyche. Allgem. Zeitschr. f. Psych. 68, H. 5. 1911.

2) $\mathrm{Zu}$ der gleichen Anschauung, wenn auch auf anderem Wege, kommt Bleuler (1. c. S. $29 \mathrm{ff}$.).

3) Wie es z. B. durch Unaufmerksamkeit zu einer typischen Kontamination kommen kann, dafür folgendes, kürzlich von mir erlebtes Beispiel: Ein junges Mädchen sagt, während sie sich vor dem Spiegel ihren Hut aufsetzt, dabei aber in ihren Gedanken mit etwas anderem beschäftigt ist: ,In Amerika ist eine Geldstrafe auf Hutnadeln verboten worden." (Kontaminiert aus: „In Amerika sind Hutnadeln verboten worden" und ,es ist eine Geldstrafe auf das Tragen von Hutnadeln gesetzt worden.")

4) Siehe Ziehen, Physiologische Psychologie. Jena 1911. S. 172ff. 
offenbar lange vor den intellektuellen vorhanden, wie das aus zahlreichen Beobachtungen der Kinderpsychologie hervorgeht ${ }^{1}$ ). Beim erwachsenen Menschen finden wir freilich fast immer Affektives an Intellektuelles gebunden und ebenso umgekehrt Intellektuelles kaum je ohne Affektives, so daß die Trennung von beiden uns oft als etwas Künstliches erscheinen mag. Das aber hängt mit der psychischen Entwicklung überhaupt zusammen, die, je höher sie ist, eine um so innigere und allgemeinere Verknüpfung der psychischen Elemente und Gruppen darstellt. Zudem besteht zwischen Intellektuellen und Affektiven, auch wenn sie gleichzeitig vorhanden sind, nicht nur keine Parallelität, sondern im Gegenteil gewöhnlich sogar ein ausgesprochener Antagonismus. Die Klarheit unserer Vorstellungen und die Intensität ihrer ,,Gefühlstöne" stehen häufig genug in umgekehrtem Verhältnis $\left.{ }^{2}\right)$. Schon das spricht gegen die Auffassung einer ,,Gefühlsbetonung " der Vorstellungen als einer Eigenschaft von ihnen. Weiter kennen wir auch Gefühlszustände ohne jede intellektuelle Grundlage. Wir reden in diesen Fällen gewöhnlich von „Stimmungen“. Es gibt ferner, wie wir besonders durch die Freudschen Untersuchungen wissen, manche psychische Mechanismen, in denen eine völlige Abtrennung des Gefühlszustandes von dem ursprünglich zugehörigen Vorstellungsinhalt stattfindet. Eine gewisse Selbständigkeit zeigen die Gefühlszustände weiter auch darin, daß sie bei den verschiedenen Menschen auch bei gleichen intellektuellen Vorgängen äußerst verschieden sind. Diese Verschiedenheit der individuellen Reaktion ist auf affektivem Gebiet, ganz im Gegensatz zum intellektuellen, so stark, daß man hier, wie Bleuler das hervorhebt ${ }^{3}$ ), ,eigentlich noch gar keinen Maßstab hat, was normal und was pathologisch ist". Auch manche klinische Beobachtungen sprechen gegen die Annahme einer ,,Gefühlsbetonung" der Vorstellungen im Sinne der Assoziationspsychologie. Insbesondere widersprechen einer derartigen Anschauung Erscheinungen, wie wir sie bei der Dementia praecox finden. Denn es läßt sich kaum vorstellen, wie gerade nur die Gefühlstöne der Vorstellungen durch einen krankhaften Gehirnprozeß geschädigt werden sollten.

Wie haben wir uns also denn die Verknüpfung des Affektiven mit den Vorstellungen zu denken? Um hierfür eine Antwort zu finden, gehen wir zunächst wieder von den Empfindungen aus und legen uns die Frage vor: Was ist eigentlich das, was wir als "Gefühlskomponente“" der Empfindungen kennen gelernt haben?

1) Derartige Beobachtungen besonders bei Preyer, Ribot, Ble uler (l. c.).

2) Also ganz entsprechend den Empfindungen, wo, wie wir fanden, der Grad ihrer Gefühlskomponente dem Grade ihrer Reproduzierbarkeit umgekehrt proportional ist.

3) Bleuler, l. c. S. 23. 
Die Auffassung der Assoziationspsychologie, den Gefühlston als eine bloße Eigenschaft der Empfindung zu betrachten und ihn also der Qualität und Intensität der Empfindungen an die Seite zu stellen, ist unhaltbar. Schon deshalb, weil es sicher auch indifferente, d. h. Empfindungen mit nur sehr geringer oder gar keiner Gefühlsbetonung gibt, und zwar, wie aus unseren früheren Darlegungen hervorgeht, speziell unter den Gesichtsempfindungen. (Denken wir etwa an eine gleichmäßige graue Fläche!). Wäre die Gefühlsbetonung der Empfindung eine Eigenschaft, wie etwa ihre Intensität, so müßte, wenn die Eigenschaft gleich Null wird, auch die Empfindung gleich Null werden, wie das ja auch in der Tat auf die Intensität, dagegen eben durchaus nicht auf die Gefühlsbetonung der Empfindungen zutrifft ${ }^{1}$ ). Weiter ist gegenüber der Anschauung von der Gefühlsbetonung der Empfindung mit Recht auch der Einwand erhoben worden, daß die Gefühlstöne der Empfindungen ihrerseits wieder eine Mehrheit von Eigenschaften haben (Qualität, Intensität, Dauer), ganz ebenso wie die Empfindungen selbst. Eine Eigenschaft in dem Sinne, wie die Gefühlstöne nach der Assoziationspsychologie es sein sollen, kann aber unmöglich selbst wieder Eigenschaften besitzen ${ }^{1}$ ). Auch schon die Tatsache, daß den Empfindungen der verschiedenen Sinnesgebiete, wie wir sahen, eine Gefühlskomponente in ganz verschiedenem Grade zukommt, macht die Lehre von dem Gefühlston der Empfindungen im höchsten Maße unwahrscheinlich.

Die Gefühlskomponente der Empfindung muß somit etwas zu den eigentlichen Empfindungen noch Hinzukommendes sein. Dabei kann es sich aber keinesfalls um irgendeine Assoziation im gewöhnlichen Sinne handeln, dazu ist die Verbindung zwischen Empfindung und Gefühlskomponente eine viel zu innige. Denn die Empfindung bildet zusammen mit ihrer Gefühlskomponente ein nur künstlich trennbares Ganze (eben erst die ganze Empfindung). Trotz der so engen Verbindung der Gefühlskomponente mit der eigentlichen Empfindung steht diese jener aber doch, wie wir sahen, als etwas durchaus Andersartiges gegenüber, ja es besteht sogar, wie wir in der obigen Reihe der Empfindungen feststellten, ein deutlich gegensätzliches Verhalten. Es zeigt also die Gefühlskomponente der Empfindung gegenüber nach Ebbinghaus' treffendem Ausdruck eine ,eigentümliche Mischung von Gebundenheit und Unabhängigkeit" "2). Ihrem Wesen nach erscheint sie jedenfalls nicht den Empfindungen, sondern ganz andersartigen Vorgängen zugehörig, die wir eben als ,affektive bezeichnen. Die an Empfindungen gebundenen, die ,,sinnlichen" und die sog. ,geistigen"

1) Vgl. dazu Stu mpf, Über Gefühlsempfindungen. Zeitschr. f. Psychol. 44, H. $1,1907$.

2) Ebbinghaus, Psychologie. Leipzig 1902. S. 542. 
Gefühle erscheinen - soweit letztere überhaupt wirklich Gefühle und nicht Vorstellungen sind - als etwas Zusammengehöriges ${ }^{1}$ ); in beiden Fällen erleben wir psychisch ganz Ähnliches, jedenfalls in beiden Fällen etwas, was sowohl von den eigentlichen Empfindungen wie den Vorstellungen durchaus wesensverschieden erscheint.

Aus alledem ergibt sich für die Art der Verknüpfung von Empfindung und Gefühlskomponente: die Gefühlskomponente muß einerseits in gewisser Weise ihre selbständigen physiologischen Bedingungen haben, andererseits müssen diese aber auch irgendwie besonders eng mit dem zusammenhängen, vermutlich sogar zum Teil zusammenfallen, was wir als die physiologischen Grundlagen der Empfindungen kennen.

Es scheint mir nun überhaupt nur eine einzige Annahme zu geben, die diese Forderung erfüllt und sich gleichzeitig auch mit allen den hier bisher dargelegten psychologischen Tatsachen vereinigen läßt: nämlich die Annahme, daß es sich um zentrale, und zwar subcorticale Miterregungen handelt. Es würden in diesem Falle also auch die eigentlichen Gefühlsvorgänge, die Affektivität, ihre Grundlage irgendwie in subcorticalen Erregungen haben. Für eine derartige Annahme lassen sich besonders folgende, zum Teil im vorangegangenen schon mehrfach berührte Tatsachen anführen.

1. Die rein affektiven Vorgänge sind etwas ihrem ganzen Wesen nach völlig Andersartiges als die psychischen Vorgänge, die wir als sicher cortical bedingt kennen, die eigentlichen Empfindungen und Vorstellungen.

2. Die Art der Verbindung der Gefühlsanteile der Empfindungen sowohl wie der Vorstellungen mit diesen ist eine ihrem Wesen nach gänzlich andere als die Verknüpfung corticaler Erregungen, die Assoziation.

3. Affektive Vorgänge sind in der Stammesreihe wie beim Einzelindividuum bereits vor den intellektuellen vorhanden; das erste, was wir in der Entwicklung der kindlichen Psyche finden, sind affektive Vorgänge, und zwar bereits zu einer Zeit, wo die Assoziationsfasern noch gar nicht ausgebildet sind.

4. Bei vielen krankhaften Gehirnprozessen, und zwar gerade bei solchen, die besonders die Hirn rinde schädigen, wie die Paralyse, die senile Demenz, erleidet meist nicht die Affektivität an sich eine Einbuße, sondern nur das ,, höhere Gefühlsleben“", d. h. die an Vorstellungskomplexe gebundene Affektivität. Die Affektivität an sich ist dagegen sogar häufig dabei erhöht (Stimmungslabilität!), weil eben die hemmenden Einflüsse gewisser Vorstellungsreihen fortfallen.

Alles dies weist also darauf hin, daß für das Zustandekommen

I) „Die Unterscheidung zwischen physischem und psychischem Schmerz hat wohl einen praktischen, aber keinen wissenschaftlichen Wert" (Ribot, 1. c. S. 63). 
dessen, was als Gefühlsanteil mancher Empfindungen einen wesentlichen Bestandteil von ihnen ausmacht, und was als Affektivität die Grundlage unseres Gefühlslebens bildet, subcorticale Erregungen von besonderer Bedeutung sind. Gleichzeitig würde die Annahme solcher subcorticaler, also zentraler Miterregungen auch dafür eine Erklärung geben, warum uns eine Trennung der doch als etwas Verschiedenes von der eigentlichen Empfindung erkannten Gefühlskomponente im Einzelfall nicht möglich ist, vielmehr unserem psychischen Erleben die eigentliche Empfindung zusammen mit ihrer Gefühlskomponente stets als ein untrennbares Ganzes erscheint. Diese Tatsache wird uns noch verständlicher, wenn wir jetzt an die Frage herantreten, welche von den subcorticalen Zentren hier wohl besonders in Betracht kommen könnten.

Das eigentümliche Verhältnis zwischen den Empfindungen und ihrer Gefühlskomponente, diese „eigentümliche Mischung von Gebundenheit und Unabhängigkeit", hat vielfach den Gedanken nahe gelegt, daß es sich hierbei um zwei Wirkungen einer Ursache handle. So meint Ebbinghaus, man müsse, um den hier zu beobachtenden Tatsachen gerecht zu werden, annehmen, daß es sich bei den Gefühlen um Nebenwirkungen derselben Ursachen handeln müsse, die den begleitenden Empfindungen und Vorstellungen zugrunde liegen; ,so daß also gleichzeitig durch Einwirkung jener Ursachen auf gewisse Gebilde des Organismus der intellektuelle Effekt und durch Einwirkung auf andere Gebilde der dazugehörige Gefühlseffekt hervorgebracht wird" 1 ).

Lassen wir zunächst einmal die Vorstellungen außer acht und beschränken wir den Satz auf die Empfindungen (wobei also unter dem ,intellektuellen Effekt" die eigentliche Empfindung, das Objektive an ihr zu verstehen wäre). Wir wissen nun, daß es die Erregung von seiten der Hirnrinde ist, die unsere Empfindungen uns bewußt werden läßt, und daß also der sozusagen intellektuelle Anteil der Empfindungen, die eigentliche Empfindung (ihr objektiver, d. h. reproduzierbarer Anteil) sicher an corticale Vorgänge gebunden ist. Wir wissen aber weiter, daß jeder Empfindung anatomisch-physiologisch eine Erregung nicht nur von spinalen und corticalen, sondern stets auch von subcorticalen Zentren entspricht. Von besonderer Bedeutung ist hier der Thalamus opticus. Denn er schiebt sich als Schaltstelle im wesentlichen für die sensiblen Bahnen, also die Hautsinnesempfindungen, zwischen Peripherie und Großhirn, durch ihn müssen alle sensiblen Reize hindurch, um zur Großhirnrinde zu gelangen. Die Annahme liegt also nahe, daß bei den subcorticalen Erregungen, die nach der oben entwickelten Anschauung die physiologische Grundlage der Gefühlskomponente der Empfindung bilden, vor allem solche im Thalamus opticus in Betracht kommen. Mit einer derartigen Annahme wird selbstverständlich

1) Ebbinghaus, 1. c. S. 542 . 
nicht etwa irgendein „Gefühlszentrum" statuiert (was, obwohl es sich ja aus dem Bisherigen ergibt, doch hier nochmals ausdrücklich hervorgehoben sei). Denn wenn auch die Gefühlskomponente physiologisch durch derartige subcorticale Erregung bedingt ist, so ist das natürlich nicht dahin zu verstehen, daß dieser Erregung direkt psychisch irgendein Gefühl entspricht; vielmehr entspricht beiden Erregungen zusammen die ganze Empfindung, die wir ja, wie wir mehrfach betonten, trotz der in ihr vereinigten heterogenen Komponenten als etwas durchaus Einheitliches und psychisch Einfaches erleben ${ }^{\mathbf{1}}$ ). Somit kann auch, wenn im subcorticalen Zentrum, im Thalamus, eine Unterbrechung der sensiblen Bahnen etwa durch einen krankhaften Prozeß gegeben ist, nichts anderes eintreten, als was eben auch sonst bei jeder Unterbrechung der sensiblen Bahnen erfolgt, daß eben die ganze Empfindung entsprechend den unterbrochenen Bahnen ausfällt. Es liegt also in der Natur der Sache, daß die hier entwickelte Theorie in dieser Beziehung durch die Tatsachen der pathologischen Anatomie weder eine Stütze noch eine Widerlegung erfahren kann.

Wir sahen, daß unsere Gefühlsvorgänge überhaupt nichts wesentlich Andersartiges sind als das, was uns bei den Empfindungen als Gefühlskomponente entgegen tritt, und da $\beta$ auch sie in subcorticalen Erregungen ihre hauptsächliche Grundlage haben müssen. Indem wir uns das anatomisch-physiologische Substrat der Empfindungen vergegenwärtigten, kamen wir dazu, unter diesen subcorticalen Erregungen für das Zustandekommen der Gefühlskomponente dem Thalamus opticus eine besondere Bedeutung zuzuweisen. Er müßte eine ähnliche dann also auch für die Gefühlsvorgänge überhaupt, für die Affektivität haben. Hier ist es nun bemerkenswert, daß wir aus klinischen Beobachtungen, vor allem der bei Thalamusläsionen häufigen mimischen Facialislähmung, wissen, daß der Thalamus opticus zu den affektiven Ausdrucksbewegungen in besonderer Beziehung steht, und daß also gewisse Psychoreflexe durch ihn vermittelt werden. Diese Tatsache paßt somit gut zu der Anschauung von einem Zusammenhange zwischen den affektiven Vorgängen und der Tätigkeit der subcorticalen Zentren. Utber die genauere Art und das Wesen dieses Zusammenhangs gibt aber auch sie keinen Aufschluß. Man könnte ja zunächst daran denken, die durch den Thalamus vermittelten Psychoreflexe in Vergleich zu setzen mit anderen Reflexvorgängen und den anderen psychischen Erscheinungen, die man auf den Reflex zurückführen kann, und sich etwa folgendes vorstellen: Von den drei übereinandergeschalteten Reflexbögen, dem spinalen, subcorticalen und corticalen, entspricht beim spinalen der direkten Reflexübertragung psychisch überhaupt

1) Von dem hier gegebenen Standpunkt aus kann man auch, wenn man so will, das Gefühl als „Funktion der Fmpfindung“ bezeichnen. 
nichts, bei dem corticalen schieben sich corticale Erregungen, d. h. bewußte Vorgänge, Vorstellungsreihen dazwischen (wir sprechen dann von „Handlungen"), den subcorticalen entspricht ein Gefühlsprozeß. Daß aber in Wirklichkeit die Verhältnisse so einfach jedenfalls nicht liegen, beweisen die Thalamusläsionen, wo eben nur der mimische Ausdruck des Affekts gestört, dieser selbst aber durchaus vorhanden ist ${ }^{\mathbf{1}}$ ).

Wenn also auch alles auf einen Zusammenhang der Affektivität mit subcorticalen Vorgängen hinweist, so ist uns doch die Art dieses Zusammenhanges heute noch ganz dunkel. Vielleicht ist die Affektivität irgendwie aus Kombinationen und Komplikationen der Gefühlskomponente der Empfindungen hervorgegangen. Denn aus dieser heraus hat sie sich jedenfalls entwickelt, und wie diese im wesentlichen auf subcorticale trregungen zurückzuführen ist, so müssen auch bei ihr ähnliche Vorgänge irgendwie eine Rolle spielen.

Da unser höheres Gefühlsleben, wie wir sahen, aus einer eigenartigen Verknüpfung der Affektivität mit Vorstellungskomplexen hervorgeht, so muß ihm nicht nur die Tätigkeit der ganzen Großhirnrinde, sondern gleichzeitig auch die subcorticaler Zentren zugrunde liegen. Es stellt somit das komplizierteste unter allen unseren psychischen Funktionen dar, womit übereinstimmt, daß es als letzte von allen sich entwickelt hat und als erste von allen geschädigt wird. Von der Affektivität selbst, die eine der ersten und primitivsten unserer psychischen Funktionen ist, wissen wir nur, daß sie in irgendeiner Weise mit der Tätigkeit der subcorticalen Zentren verknüpft sein muß. Eine genauere Einsicht in ihr Wesen und ihre physiologischen Bedingungen haben wir nicht gewinnen können.

Wenn wir aber so auch das Gefühlsproblem nicht lösen können, so glauben wir doch wenigstens gezeigt zu haben, auf welchem Wege seine Lösung $\mathrm{zu}$ suchen ist. Weder indem man die mit Gefühlszuständen verbundenen Vorstellungskomplexe bis ins einzelne zergliedert, noch indem man die sie begleitenden körperlichen Erscheinungen analysiert, wird man dem eigentlichen Wesen der Gefühlszustände jemals irgendwie näher kommen. Vielmehr wird das nur dadurch möglich sein, daß man zunächst von den Empfindungen ausgeht, da uns bei diesen die einfachsten Formen von affektiven Vorgängen entgegen treten, und daß man erst einmal die Gefühlskomponente der Empfindungen zum Gegenstand genauerer Untersuchungen macht.

1) Ich hatte Gelegenheit, an der Greifswalder Nervenklinik eine derartige Beobachtung zu machen, wo bei einem (zur Sektion gekommenen) Fall von Thalamustumor eine ausgesprochene mimische Facialislähmung bestand, dic Affektivität aber auch bei der besonders darauf gerichteten Untersuchung sich als ganz normal erwies. 